\title{
Retrospective Analysis of the Management of Kaposi's Sarcoma: Single-Center Experience
}

\author{
R EL Hazzaz*, K Oualla, K Darif, H Erraichi, S Berrad, L Nouiyekh, L Amaadour, Z Benbrahim, S \\ Arifi and N Mellas \\ Department of Medical oncology, Morocco
}

*Corresponding author: R EL Hazzaz, Department of Medical oncology, University Hospital Hassan II Center Fez, Morocco.

To Cite This Article: R EL Hazzaz*, K Oualla, K Darif, H Erraichi, S Berrad, et al., Retrospective Analysis of the Management of Kaposi's Sarcoma: Single-Center Experience. Am J Biomed Sci \& Res. 2021 - 13(4). AJBSR.MS.ID.001881. DOI: 10.34297/AJBSR.2021.13.001881.

Received: 㯺 May 10, 2021; Published: 㘹 July 07, 2021

\begin{abstract}
Introduction: Kaposi's sarcoma (MK) is most likely caused by human herpes virus type 8 (HHV-8) which develops particularly in individuals infected with HIV. This disease causes purplish or brownish tumors on the skin. Other organs can be affected, such as the mucous membranes, and the viscera. The goal of our study is to describe the epidemiological, clinical and therapeutic profile of this disease. Chemotherapy has big interest in Kaposi sarcoma.

Methodology: This is a retrospective study conducted in the Medical Oncology Department at University Hospital Hassan II Center of FES about 13 patients over a period of 9 years from January 2010 to December 2018 to illustrate the service's experience in the management of Kaposi's sarcoma.

Results: The annual incidence of Kaposi disease in our department was very low, not exceeding three cases per year during the period studied. We have noted a male predominance with a sex ratio of 3,33. In our study, classical Kaposi disease was the most common form and presented in almost all patients with skin lesion in the form of papulonodules or purplish erythematous-angiomatous plaques. At the time of diagnosis, two patients had stage III and eleven patients had stage IV with visceral involvement according to the KRIEGEL classification. Chemotherapy was the standard treatment for almost all of our patients, other treatments such as surgery or radiotherapy were used in localized disease. The median progression-free survival of Kaposi disease treated in our department is 12 months and the average global survival of the Kaposi sarcoma extends to $52.95 \%$ over a 20 -month follow-up.
\end{abstract}

Conclusion: In our department, we mostly found the classic form of Kaposi disease. Chemotherapy takes an essential place in the management of advanced cases. Better knowledge of signaling voices allowed the discovery of other therapeutic targets that may be promising in the future.

Keywords: Sarcoma; Kaposi; Chemotherapy; Radiotherapy

\section{Introduction}

Kaposi's sarcoma is a tumor associated with infection with the human herpes virus 8 (HHV8) which develops particularly in individuals infected with HIV. This disease causes the appearance of purplish or brownish tumors on the skin. Other organs can be affected, such as the mucous membranes, and the viscera.

KS includes four epidemiological forms: classic KS, endemic $\mathrm{KS}$, iatrogenic KS developing in immunocompromised individuals after organ transplantation, for example, and AIDS-related Kaposi sarcoma or AIDS-KS.

The goal of our study is to describe the epidemiological, clinical and therapeutic profile of this disease. The management of KS is multidisciplinary between medical oncologists, radiotherapists, dermatologists, surgeons and pathologists, hence the interest in discussing cases in multidisciplinary consultation meetings. Chemotherapy has big interest in Kaposi sarcoma. The prognosis is quite poor and depends on the location of the tumor, the immune system and the systemic symptoms.

\section{Patients and Methods}

This is a retrospective descriptive and analytical study including patients with kaposi sarcoma in the medical oncology department at the Hassan II University Hospital Center over 9 years from January 2010 to December 2018, with a biopsy confirming the 
diagnosis and having done additional examinations to establish a classification and an extension assessment, and excluding patients who don't respond to the inclusion criteria or have incomplete data.

The data was collected through the archived paper files and the computer system of the Hassan II University Hospital (Hosix.net) to be recorded on an exploitation sheet and analyzed by the software (Microsoft office Excel) and the SPSS21 software of epidemiology.

Quantitative variables were expressed as average \pm standard deviation and qualitative variables as percent. Survival analysis is established using the Kaplan-Meir method.

\section{Results and Discussion}

Thirteen files of patients with Kaposi's disease were collected in the patient register of the medical oncology department at the Hassan II Hospital Center in Fez. The analysis of the data collected led to the following results:

\section{Epidemiological data}

The male sex was predominant with 10 men $(76.9 \%)$ for 3 women $(23.1 \%)$, the patients had a median age of 65 years with a standard deviation of 18.315 and extremes ranging from 27 to 87 years. All of our patients are Moroccan, none of the patients had a sub-Saharan origin.

Kaposi's disease is particularly very rare, this is shown by the very minimal annual incidence of this cancer at the medical oncology department of Fez.

\section{Clinical study}

In our study, the most frequent clinical form was classical Kaposi disease with a percentage of $84.6 \%$ in eleven HIV negative patients. Patients were evaluated according to performance status based on the OMS classification, which is used to rate patients according to their activity level. The assessment is established upon confirmation of the diagnosis. In our series, two patients had an OMS of 0 , ten patients had an OMS of 1 and another patient had an OMS of 2. Kaposi's disease in our series was not associated with significant functional signs, the two signs found are pruritus with a percentage of $23.1 \%$ followed by pain with a percentage of $15.4 \%$. On dermatological examination, papulonodules and purplish erythematous-angiomatous plaques are the lesions most often found in our patients (Figure 1-3) ${ }^{1}$.

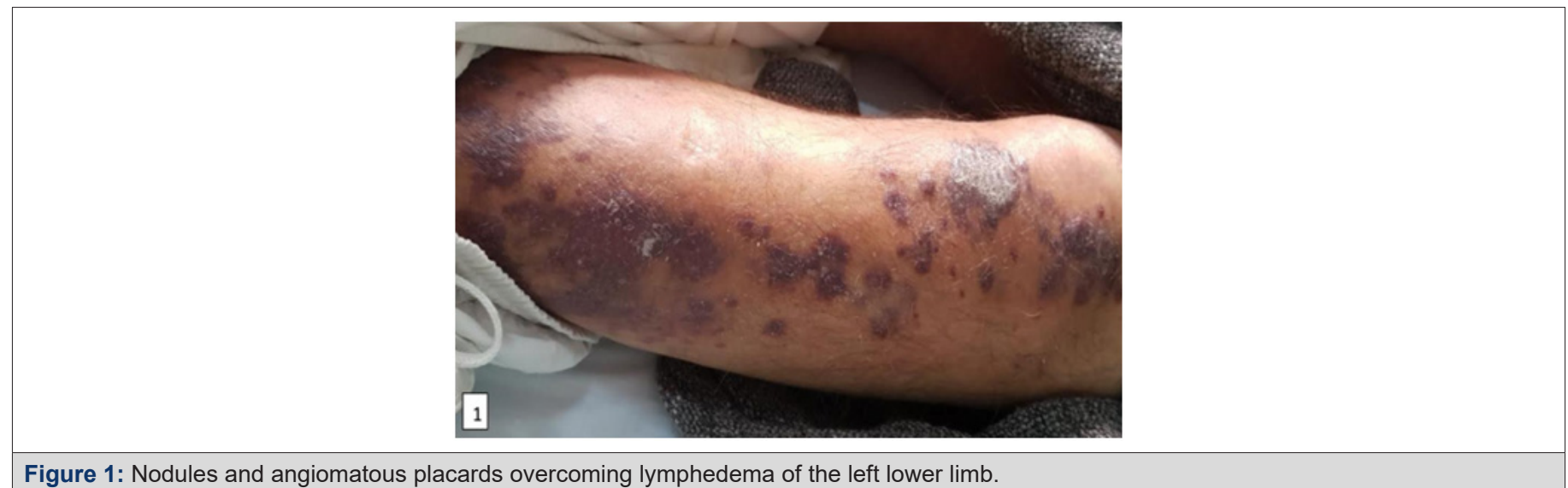

Figure 1: Nodules and angiomatous placards overcoming lymphedema of the left lower limb.

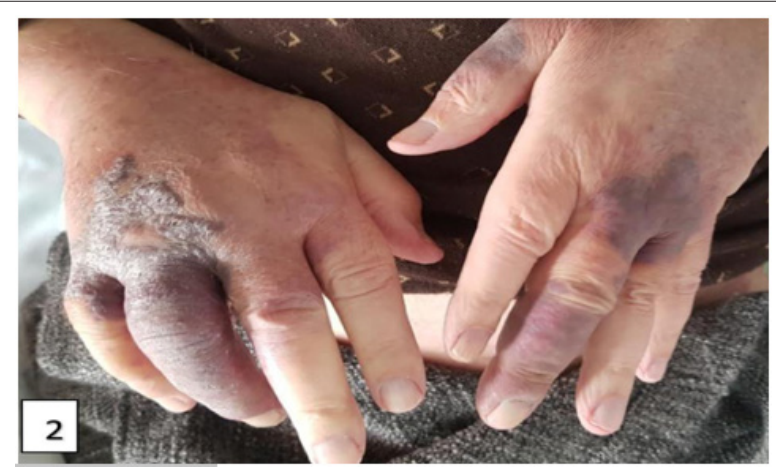

Figure 2: Involvement of the upper limbs.

${ }^{1}$ Pictures taken at the medical oncology department, CHU Hassan II in Fez. 


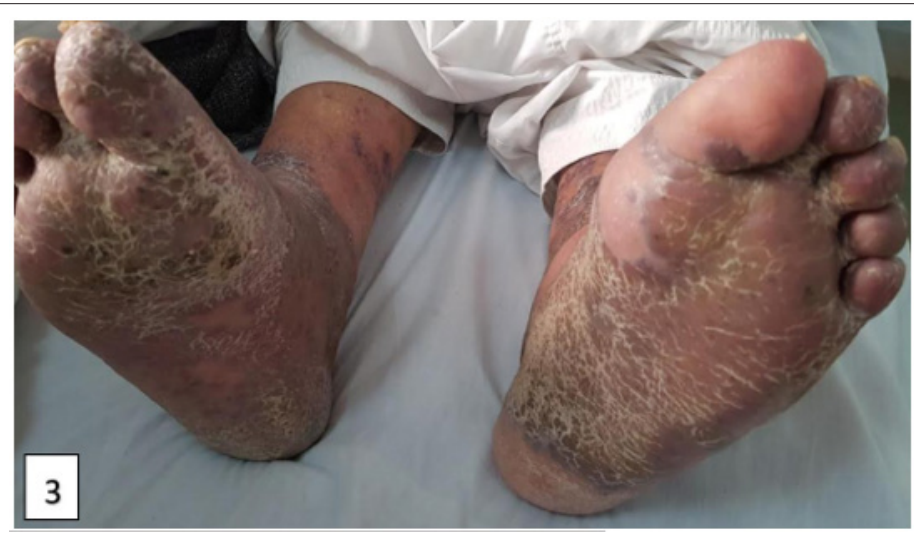

Figure 3: Ulcerated infiltrated placard of the soles of the feet.

The most frequent localization of Kaposi's sarcoma was the foot with a percentage of $53.8 \%$ in seven patients, followed by the hand with a percentage of $38.5 \%$ in 5 patients then the leg with a percentage of $23.1 \%$ in 3 patients. The mucous membranes were also affected by Kaposi sarcoma, one patient had involvement of the oral mucosa and another patient had involvement of the genital mucosa (penis).

\section{Histology}

All patients had a positive biopsy and anatomopathological examination in favor of Kaposi sarcoma. Two patients have done immunohistochemistry that came back positive for anti CD34 and anti HHV8 antibodies.

\section{Paraclinical study}

The most common used radiological examination was the thoraco-abdominopelvic (TAP) scan, which was performed in all patients to search for secondary locations. Among the lesions found, the most frequent were pulmonary involvement in ten patients (76.9\%) mainly represented by pulmonary micronodules. FOGD (eso-gastro-duodenal fibroscopy) is performed in all our patients and identified lesions in six patients (46.2\%), two of whom were in favor of gastroduodenal kaposi lesions confirmed on biopsies.

\section{Staging}

In our series, two patients had stage III (15.4\%) and eleven patients had stage IV with visceral involvement (84.6\%).

\section{Treatment}

Chemotherapy was the standard treatment for almost all of our patients, other treatments such as surgery including cryoablation in one patient and surgical treatment of a plantar lesion in another patient and radiotherapy were used to improve local disease control. First-line chemotherapy was administered in almost all of our patients except for one patient with a localized stage. The chemotherapies used were paclitaxel, bleomycin, doxorubicin, ABV protocol and AV protocol, while second-line chemotherapy was based primarily on paclitaxel.

The results of the response after treatment were marked by the efficacy of systemic chemotherapy with paclitaxel, whether in the first or second line of therapy. The side effects observed during chemotherapy were mainly fatigue and neutropenia.

\section{Survival analysis}

The median progression-free survival for kaposi disease treated in our department is 12 months. On a follow-up of 20 months, the mean overall survival of the disease is extended to $52.95 \%$, the 5-year survival reaches $66.7 \%$.

\section{Discussion}

With around 42,000 new cases and 20,000 deaths, Kaposi's sarcoma (SK) is a relatively rare cancer worldwide but is endemic in several countries in southern and eastern Africa and believed to be the leading cause of incidence and cancer mortality in 2018 in Malawi, Mozambique, Uganda and Zambia.

Kaposi's sarcoma is grouped into four epidemiological forms [1]: classic SK affecting elderly men of Mediterranean or Eastern European Jewish descent, endemic SK, existing in parts of central and eastern Africa, described well before the HIV pandemic and often affecting children with disseminated lymphadenopathy [24], iatrogenic KS, developing in immunocompromised individuals after organ transplantation, for example [5], and AIDS (acquired immune deficiency syndrome) related kaposi sarcoma or AIDS-SK. In the western world, AIDS-SK mainly affects gay men infected with HIV. However, in Africa, since the spread of HIV, the KS epidemic has become more common in both sexes, with a dramatic decline in the ratio of males to females, particularly in East Africa [6]. A North American study published in 2008 was interested in comparing the incidence of SK associated with AIDS and classical kaposi disease, this study included 438 clinically and histologically confirmed patients. Taking into consideration ethnic diversity and 
racial distribution; 38\% of patients with classical KS are Caucasian Americans and 11\% are of origin Mediterranean [7].

Kaposi's sarcoma (SK) is a vascular lesion of low malignant potential associated with infection with human herpesvirus 8 (HHV8) [8]. MKC is a multifocal, often multicentric condition. It is usually limited to the skin, but can sometimes be localized in the mucous membranes, lymph nodes or viscera [9].

Overall, 4 forms of kaposi sarcoma are described [10]:

1. "Classic" Kaposi's disease (MKC): The basic lesions are macules, erythematous and purple plaques that gradually infiltrate. These lesions do not disappear with vitropression especially when they are old and readily take on an ecchymotic, hemorrhagic or pigmented appearance [8]. The lesions are often bilateral, predominantly in the extremities, especially in the lower limbs; bullous lesions can be observed but are exceptional [8-11].

2. Endemic kaposi disease: Ocular and periorbital involvement is very suggestive [12]. Sometimes comparable in appearance to classic KD (nodular form), endemic KD more often produces aggressive infiltrating tumor lesions locally or disseminated, with cutaneomucosal and visceral damage with a very poor prognosis.

3. Epidemic Kaposi disease: Skin involvement is present in over $90 \%$ of affected subjects with frequent lymph node involvement. In almost half of patients with Kaposi's sarcoma, there is damage to the digestive tract, which almost always occurs in association with skin damage. The esophagus, stomach, duodenum, colon, and rectum can all be affected. Usually, the involvement of the digestive tract is asymptomatic; however, in more advanced stages, lesions in the upper digestive tract can cause digestive symptoms [12].

4. Kaposi's disease(KD) afteriatrogenicimmunosuppression: Complications after organ transplants are frequent and varied, mainly related to induced immunosuppression. The risk of cancer is greatly increased in transplant recipients, more particularly viro-induced cancers such as Epstein-Barr virus (EBV) lymphoproliferations, cancers associated with human papillomaviruses. KD accounts for $11.2 \%$ of neoplasias, and appears faster than most other cancers, with the average time between transplantation and onset of KD being 20 months [13].

The definitive diagnosis of Kaposi's sarcoma is based on histological examination of biopsy samples, even though the disease is easily recognized on clinical examination. In general, it is not difficult to obtain samples, since most patients have skin lesions in which it is easy to take a biopsy. Regardless of the stage of the disease, the spindle cell represents the kaposian cell. The histological features appear to be the same whether it is classical Kaposi disease or associated with HIV [14]. The KRIEGEL classification, implemented since 1983, is the most used and the simplest for staging all forms of KD except that associated with HIV infection, in order to assess the severity of KD and thus allows guide the therapeutic strategy [15].

Systemic treatments are reserved for widespread and locally aggressive KS: the recommended first-line agents are pegylated liposomal doxorubicin (PLD) and paclitaxel. In classical Kaposi's sarcoma, low dose PLD or interferon alfa are the recommended first-line agents in younger patients. In AIDS-related kaposi sarcoma, combination antiretroviral therapy is the first treatment option; specific systemic therapy is only necessary in extensive disease and in the prevention and treatment of immune reconstitution inflammatory syndrome. In post-transplant kaposi sarcoma, reduction of immunosuppressive therapy and switching of rapamycin inhibitors (m-TOR) are used [16].

Local therapies are offered in cases of poorly progressive forms. Cryotherapy is used for lesions less than $1 \mathrm{~cm}$ in diameter, for larger lesions cryosurgery has more followers than intralesional chemotherapy. The use of radiotherapy is only recommended in the event of failure of these treatments. chemotherapy, in particular based on Bleomycin, are used in the rapidly evolving cutaneous-mucous forms or systemic forms which do not rapidly endanger the vital prognosis. Liposomal anthracyclines, ABV type polychemotherapy (adriamycin, bleomycin, vincristine) or, probably better, Taxanes will be reserved for forms which have escaped the aforementioned treatments or in the event of dyspneic pulmonary locations [17]. In the absence of antiretroviral treatment, the progression is rapidly progressive towards a multisystem form, associated with opportunistic infections that should be systematically sought out [10]. In the classic form of Kaposi disease, of slow evolution and predominantly cutaneous determinism, localized for a long time and which mainly affects elderly subjects, the vital prognosis is only rarely threatened in the short or medium term in a direct way by the kaposi disease. The treatment will then aim to correct functional or aesthetic damage.

\section{Conclusion}

Kaposi's disease (or Kaposi's sarcoma) can be defined as a mesenchymal proliferative process involving cells of the blood and lymphatic systems, which is induced by viral growth factors, in particular human herpes virus type 8 interleukin 1 (HHV8). It is a rare neoplasia of the endothelial cells. Multiple therapeutic choices are offered for the treatment of kaposi disease. The type of treatment requires an individualized approach based on the extent and location of the lesions, the patient's wishes, the presence of symptoms associated with the tumor, the presence of associated pathologies and the patient's tolerance to treatment. In our series, the chemotherapy used resulted in a considerable improvement in 
survival in the majority of patients. Further prospective, multicenter studies with larger sample sizes are needed to better optimize the treatment and management of patients with Kaposi's sarcoma.

\section{References}

1. RC Gallo (1998) The Enigmas of Kaposi's Sarcoma. Science 282(5395): 1837-1839.

2. A G Oettle (1962) Geographical and racial differences in the frequency of Kaposi's sarcoma as evidence of environmental or genetic causes. Acta Unio Int Contra Cancrum 18: 330-363.

3. Slavin G, Cameron HM, Forbes C, Mitchell RM (1970) Kaposi's sarcoma in East African children: A report of 10 cases. J Pathol 100(3): 187-199.

4. Bhagwat GP, Naik KG, Sachdeva V, Bhushan V (1980) Disseminated lymphadenopathic Kaposi's Sarcoma in zambian children. Med J Zambia 14(4): 61-63.

5. Siegel JH, Janis R, Alper JC, Schutte H, Robbins L, et al. (1969) Disseminated visceral kaposi's sarcoma: appearance after human renal homograft operation. JAMA 207(8): 1493-1496.

6. HR Wabinga, DM Parkin, F Wabwire-Mangen, JW Mugerwa (1993) Cancer in Kampala, Uganda, in 1989-91: Changes in incidence in the era of aids. Int J Cancer 54(1): 26-36.

7. Hiatt KM, Nelson AM, Lichy JH, Fanburg-Smith JC (2008) Classic Kaposi sarcoma in the United States over the last two decades: a clinicopathologic and molecular study of 438 non-HIV-related Kaposi Sarcoma patients with comparison to HIV-related Kaposi Sarcoma. Mod Pathol 21(5): 572-582.
8. Grayson W, Pantanowitz L (2008) Histological variants of cutaneous Kaposi sarcoma. Diagn Pathol 3: 31

9. CISMeF (2020) Kaposi's sarcoma MeSH descriptor. CISMeF, Rouen University Hospital [Internet].

10. Aubry PP (2019) Maladie de Kaposi Actualités.

11. Manifestations dermatologiques chez les sujets IB POSITIFS AU cHNYo (Aspects Cliniques Et Épidémioloeiiques). Universite De Ouagadougou, Africa.

12. Taylor JF, Templeton AC, Vogel CL, Ziegler JL, Kyalwazi SK (1971) Kaposi's sarcoma in Uganda: A clinico-pathological study. Int J Cancer 8(1): 122-135.

13. Danpanich E, Kasiske BL (1999) Risk factors for cancer in renal transplant recipients. Transplantation. 68(12): 1859-1864.

14. Chor P, Santa Cruz D (1992) Kaposi's sarcoma. J Cut Pathol 19: 6-20.

15. Iscovich J, Boffetta P, Franceschi S, Azizi E, Sarid R (2000) Classic Kaposi sarcoma: epidemiology and risk factors. Cancer 88(3): 500-517.

16. Lebbe C, Garbe C, Stratigos AJ, Harwood C, Peris K, et al. (1990) Diagnosis and treatment of Kaposi's sarcoma: European consensusbased interdisciplinary guideline (EDF/EADO/EORTC). Eur J Cancer 114: 117-127.

17. Ronchese F (1958) Kaposi's sarcoma; an overlooked essay of 1882. AMA Arch Dermatol 77(5): 542-545. 